Caligrama, Belo Horizonte, v. 25, n. 3, p. 9-16, 2020

\title{
Les littératures latino-americaines d'expression française : vues du Brésil ou « la malédiction de l'anachronisme » d'Octavio Paz
}

L'impérialisme français a toujours été extrêmement efficace dans le domaine culturel. La France, nation conquérante, a toujours utilisé sa force militaire et parallèlement sa langue comme outil de colonisation mentale. C'est son power soft qui a connu un succès retentissant jusqu'à nos jours dans les soi-disant « anciennes colonies » du Nouveau Monde, et même dans les départements et territoires d`outre-mer. L'un des exemples de power soft est, au-delà de l'implantation et de l'utilisation de la langue française comme langue de communication, de diplomatie, d'expression de l'identité, l'éclat et le prestige accordés à la langue française qui phagocyte l'imaginaire des poètes et prosateurs latino-américains et brésiliens et, dans leur sillage, les chercheurs. « Parler une langue, c'est assumer une culture ", rappelait Fanon dans son premier chapitre de Peau noire, masques blancs : la langue française sert sous ces latitudes à rassembler le social, et aussi à manifester le culturel, l'imaginaire, le politique. Elle cristallise les réalités imaginaires d'une identité locale qui n'a presque pas d'expressivité si elle se fait en dialectes locaux - les créoles et les langues indigènes, par exemple, aux Antilles et en Guyane. L'utilisation du français pour écrire est un acte démocratique - le choix de s'exprimer en français pour être davantage lu, surtout par ceux qui, en fait, ont besoin de connaître et d'être en contact avec des réalités locales fictives ou non-fictives. C'est par ce biais que les littératures de langue française prennent racine en Amérique du Sud. Des récits écrits dans une langue de communication.

Le binôme langue / nation est abandonné pour donner naissance à un lieu de parole ou l'expression d'une identité franco-brésilienne ; la langue française devient une langue d'accueil pour elle, ainsi que pour les autres identités latino-américaines et leurs cultures respectives.

Ce rapport entre démocratie, acceptation et altérité de la langue française s'observe dans tous les territoires où le français était, et est encore, présent. Appartenant à l'Amérique latine, il y a les « Antilles 
françaises ", la Martinique et la Guadeloupe, la République d'Haïti et le troisième département d'outre-mer, la Guyane française. Si ces régions (à l'exception d'Haïti) sont encore des territoires français, leurs dynamiques sociales, politiques et culturelles ne sont encore trop souvent mises en valeur que lorsqu'un agent culturel part vivre en métropole. Ceci se voit avec la diaspora haïtienne au Canada, en France, en Suisse et en Belgique francophone; dans le cas des territoires français, cela est constaté lorsque des personnes s'installent dans l'Hexagone - comme Aimé Césaire, Édouard Glissant, Maryse Condé, entre autres.

Tout autre est le cas de figure des littératures, dites latinoaméricaines d'expression française, qui ne sont pas produites dans leurs territoires réels, mais dans leurs nations imaginées. Cela nous amène en réalité à nous demander dans quelle mesure une littérature de la diaspora est-elle une littérature nationale, locale ou régionale ? Il n'existe pas de consensus à ce sujet à ce jour. Cependant, il faut se demander si les textes ne sont pas non plus rédigés dans une Amérique latine francophone rêvée, ancrée dans un même espace linguistique, celui du français exporté aux Amériques.

Trois aspects de cette francophonie latino-américaine et brésilienne méritent d'être soulignés. Il s'agit d'aspects bien établis dans le domaine littéraire : l'histoire, la théorie et la critique.

L'historiographie et l'histoire littéraire n'accordent encore unilatéralement de l'importance à une œuvre que lorsque celle-ci est éditée à Paris, " nombril du monde " (selon l'expression du poète guyanais, Léon Damas). Autrement dit, si l'ouvrage est accessible dans les librairies parisiennes, il peut être lu, critiqué et diffusé sous ces latitudes hispanophones et lusophones. Dans le meilleur des cas, remporter quelques Prix littéraires prestigieux aide énormément à sa diffusion dans leurs pays d'origine. Sinon, l'œuvre littéraire reste invisible et est considérée comme pratiquement inexistante. De plus, si l'œuvre est rédigée en " bon français », elle devient rapidement un « classique », une entrée dans le « canon » de la Littérature francophone. Autrement dit, le lieu d'édition et le critère d'un Français standardisé valent encore de critères d'excellence. Il est clair qu'à l'orée du second quart du XXI ${ }^{\mathrm{e}}$ siècle, il est temps de modifier cela. Grâce à Internet, cette révolution commence à apparaitre : la révolution numérique modifie de fond en comble la « fabrique de la littérature », qu'elle soit francophone, anglophone, ou toute autre littérature europhone. 
Une deuxième idée pernicieuse et persistante de ce côté du sous-continent américain, mais également dans les zones limitrophes caribéennes et guyanaises, est le préjugé à l'égard d'ouvrages de fiction en français produits dans cette région. En Amérique latine et au Brésil, nous sommes nombreux à considérer que la production latinoaméricaine francophone d'auteurs métis, afrodescendants, voire blancs, risque d'être inférieure. Des générations entières, jusqu'aux années 80 du siècle dernier, ont été « formatées » de sorte à cultiver l'idée que les Belles Lettres françaises (parisiennes et provinciales) sont supérieures, aussi par le style, l'intrigue romanesque, que la structure narrative. Qu'un « monstre sacré » de la littérature colombienne, Gabriel García Márquez promulgue lui-même l'absence d'une tradition en la matière, idée reprise et confirmée par son compatriote Juan Gabriel Vásquez, n'arrange rien. Vivant entre Barcelone et Bruxelles, l'écrivain Vasquez analyse ce complexe comme une faille des régimes dictatoriaux qui ont sévi dans cet hémisphère. Dans « Écrire au présent. Trouver des poèmes dans le journal », Vásquez ${ }^{1}$ cite encore Márquez qui mit le doigt sur le problème d'une absence de tradition romanesque et donc d'un retard sur la formation d'un canon, des doutes quant à la capacité expressive. L'émergence d'une littérature " postcoloniale », concept combien polémique pour cette vaste région peine à s'imposer. Le Prix Nobel péruvien Mario Vargas Llosa employa à son tour «pittoresque », « provincial » et « anachronique » comme épithètes pour les littérateurs latino-américains des années 1950. C'est paradoxalement à un autre Prix Nobel latino-américain que nous empruntons le concept du sous-titre « la malédiction de l'anachronisme » : le poète mexicain Octavio Paz évoqua dans son discours de réception à Stockholm (PAZ, 1991) la distance temporelle qui l'a toujours séparée du Centre, Paris. Pourtant c'est au Brésil qui est né le « Manifesto Antropófago » (1928), le Manifeste de l'anthropophagie littéraire »d'Oswald de Andrade. Malgré cela, c'est encore à Paris que ces auteurs du boom ont connu le succès. Afin d'accéder à la modernité, de devenir contemporains de tous les hommes

\footnotetext{
${ }^{1}$ Écrivain en Résidence à Passa Porta, invité en 2014 à l'Université d'Anvers où il développa «La novela de la violencia ». Dans son roman Le Corps des ruines, il reprend le massacre des Colombiens innocents de la « United Fruit Company » (présent dans Cent ans de solitude de Marquez) et la longue lutte armée opposant le FARC au peuple. Passa Porta (2015, p. 124).
} 
et faire cesser la malédiction de l'anachronisme, il leur fallut le détour par Paris.

Enfin, si la littérature francophone continue à être étudiée en Amérique du Sud et au Brésil, ce sont encore et toujours les mêmes auteurs, le même " canon ", ainsi que les mêmes théories. C'est de cette impasse que le présent numéro de Caligrama cherche à sortir. Dépasser ce constat, afin de décoloniser dans l'hémisphère sud un dernier fâcheux revers, qui est celui de projeter sur ces corpora poétiques et prosaïques des schémas et modèles venus de l'extérieur qui ont tous homogénéisé ces textes et ont aplati les spécificités.

En effet, ces théories sont considérées comme des grilles d'analyses cent pour cent fiables, alors qu'elles devraient être remises en cause, affinées pour les corpora brésiliens et latino-américains.

On peut citer comme exemple la créolité, défendue par Jean Bernabé, Raphaël Confiant et Patrick Chamoiseau comme l'idéal culturel (et littéraire) de l'homme antillo-guyanais. Or, c'est un constat que la créolité se développe aux Antilles et en Guyane ; cependant, si aux Antilles la question du créole est la composante majeure de l'identité, en Guyane française, la créolité est une composante aussi importante que « l'amazonité » et la « sudaméricanité ». L'homogénéisation d'une théorie pour limiter un biais identitaire finit par limiter, voire étouffer, les différences entre les littératures locale et régionale. Ceci, en partie, peut expliquer pourquoi on parle beaucoup de littérature antillo-guyanaise et pas de littérature guyanaise, littérature martiniquaise, littérature guadeloupéenne. C'est comme si l'Antillo-Guyanais recouvrait déjà trois littératures séparées (il y a littérature antillaise, d'un côté - englobant la Martinique, la Guadeloupe - de l'autre côté, littérature guyanaise, parente pauvre des lettres francophones d'Amérique). Cependant, lorsqu'on regarde les anthologies et les collections, on sait peu de choses sur la littérature guyanaise. Le terme "Antillo-Guyanais » semble-t-il une excuse pour ne pas étudier chaque littérature particulière en détail ? Ou pour dissimuler que la littérature guyanaise demeure la parente pauvre de cet hémisphère francophone aux Amériques ? Enclave sur le continent américain et courroie entre le Nord et le Sud, la "guyanité » est à tort délaissée par le « centrisme parisien ». Territoire français d'outre-mer, elle se vit comme un état clivé, sans autonomie ou alors si peu, vu que les députés guyanais, qui s'irrite Damas, sont... Martiniquais, et qu'elle est « une Sarre africaine » (Voir DAMAS, 2003, p. 78-79). Aux yeux de Léon 
Damas, Rue Oudinot privilégia sur plusieurs domaines les Martiniquais au détriment des Guyanais, tant Paris regarde cette enclave culturellement et ethniquement « entre deux eaux », un peu au même rang que le Bélize, la Colombie, le Costa Rica... qui sont des états indépendants en Amérique du Sud. Pour arrimée qu'elle soit politiquement et économiquement aux îles, la Guyane est soudée à l'Amérique latine et sa littérature, si elle veut être représentative, devrait dès lors y chercher son ancrage. Et c'est pourtant un Guyanais qui prit soin d' " anthologiser » ces littératures émergentes et postcoloniales dans un épais numéro de Présence Africaine. Léon Damas accentue la richesse des nouvelles générations, qu'elles soient d'expression française, anglaise, américaine, portugaise, brésilienne, espagnole, néerlandaise. C'est là qu'il se démarque de Glissant, malgré tout, et quoi qu'il prétende, monolingue. Pourfendant la balkanisation au sens glissantien, l'anthologiste et cofondateur de la négritude regroupe en 1966 les littératures de la diaspora africaine du continent, de l'océan Indien et de Madagascar aux Amériques, sans passer par le Brésil, enclave souvent exclue des panoramas. Bref, il cherche à cartographier les Guyanes, singulières et multiples, dans un faisceau de Lignes afrodiasporiques :

C'est que, pour avoir retenu le message lancé par Langston Hughes, et qui devait être repris tour à tour par Palès Matos, à Porto-Rico, Nicolas Guillén à Cuba, Jacques Roumain à Port-auPrince, Claude McKay, à la Jamaïque, Cabral, à Saint-Domingue, Solano Trindade au Brésil, Debrot à Curaçao, et Albert Helman, à [sic] Suriname. Et last but not least, par Césaire, à la Martinique, par Senghor au Sénégal, et par moi-même dans la mesure où quelque haïssable que soit le moi - je ne pouvais avec mon pays, la Guyane, rester sourd à ce langage tambouriné dans la nuit, les jeunes Afro-Américains du Nord, du Centre et du Sud, poursuivent d'un pas résolu et ferme le chemin tracé (DAMAS, 1966, p. 354).

Si l'on veut en finir avec l'homogénéisation des dites littératures dans une échelle planétaire, Damas avait compris qu'il fallait une démarche résolument comparatiste afin que ces différentes aires communiquent. Quant aux lettres francophones, elles continuent de souffrir du fait que la littérature française est fixée au sommet des textes, bien que le Manifeste pour une littérature-monde (LE BRIS et al., 2007) (en français) ait voulu inverser cette perspective. Les textes en français issus d'autres cultures et d'autres régions ex-colonisées ou non par la 
France, sont considérés comme des altérités intéressantes, mais ils ne sont pas suffisamment considérés dans leur singularité, ni abordé avec des théories et des outils critiques nés dans la mouvance de la décolonialité, tournant latino-américain initié par le groupe $« \mathrm{M} / \mathrm{C} / \mathrm{D}^{2} »$ autour de, entre autres, Walter Mignolo. ${ }^{3}$

L'état des lieux («the state of the arts ») dans les départements de littérature francophone à Rio de Janeiro comme à Bogotá affiche qu'on commence à peine à découvrir cette nouvelle approche décoloniale. On peut même comprendre que l'homogénéisation soit un fort mouvement de la critique et de la théorie littéraire pour valoriser et trouver la place de ces littératures, au moins au début. Cependant, à force de maintenir cette hégémonie, on empêche l'indépendance des littératures régionales et la construction de symboles, mythes et principes théoriques propres à la transmission; en plus de repousser l'approfondissement des auteurs dehors du canon, de détails temporels et stylistiques de ces littératures par les biais diachroniques et synchroniques.

Dans une certaine mesure, la critique littéraire liée aux littératures francophones d'Amérique latine s'enthousiasme de cette homogénéisation. D'une part, la critique se contente de ce qui a été déjà tracée dans cette littérature et, d'autre part, il semble y avoir une tendance à une reproduction théorique exhaustive qui perdure depuis longtemps. En outre, les textes critiques et théoriques sur les œuvres, pour la plupart, sont encore liés au méridien de Paris, selon l'expression célèbre de Pascal Casanova (2002), une manière de valider ce type d'étude en Amérique latine. Même les auteurs francophones hors des territoires francophones (Isidore Lucien Ducasse - le Comte de Lautréamont [uruguayen]; Sergio Kokis [brésilien] ; Hector Bianciotti [argentin], Santiago Amigorena [argentin], Miguel Bonnefoy [vénézuélien] pour n'en citer que quelquesuns, toutes générations confondues) ont peu de voix et de place dans des études littéraires en Amérique latine et au Brésil. Ils n'apparaissent

\footnotetext{
${ }^{2}$ Le projet ou groupe Modernité/ Colonialité / Décolonialité (M/ C/ D) se compose de plusieurs théoriciens latino-américains tels comme : Walter Mignolo, Santiago Castro-Gómez, María Lugones, Nelson Maldonado Torres, Catherine Walsh, Ramón Grosfoguel, Arturo Escobar, d'entre autres, qui travaillent sur une pensée transnationale et transgénérationnelle à propos de la colonisation du pouvoir en plusieurs domaines, y compris la connaissance - le colonialisme de savoir.

${ }^{3}$ Lire à ce propos Mingolo (2015). Le dernier chapitre s'intitule : " Prolégomènes à une grammaire de la décolonialité ».
} 
pas dans la critique journalistique, ni dans les programmes des cours de français aux universités latino-américaines, encore moins dans les articles d'analystes littéraires dans des revues universitaires consacrées aux études francophones.

Il reste donc, on le voit bien, encore du chemin à parcourir pour décoloniser les études francophones dans cette vaste région lusophone frontalière de l'Amérique hispanophone, francophone, voire néerlandophone tant il est vrai que le Surinam manque généralement au rendez-vous comparatif, partageant pourtant le même " passé décomposé ». Les idéaux de la langue et de la culture françaises hantent encore les études littéraires de l'expression française en Amérique latine. Au Brésil, le manque de connaissance de notre « voisine française » la Guyane, en dit long sur la façon dont les études ont été menées, toujours liées à la culture française.

Ainsi, ce numéro spécial s'impose comme un espace de dialogue, d'union et de cartographie de la recherche et des chercheurs nationaux et internationaux qui pensent à la fois à la production latino-américaine de l'expression française et à contribuer à la critique des études littéraires sur la littérature francophone locale.

Ce numéro spécial permet de dégager quatre caractéristiques :

1 - les classiques de la pensée critique sur les littératures antillaises (Patrick Chamoiseau, Édouard Glissant et Aimé Césaire) apparaissent encore comme un point d'homogénéisation de la réflexion et des déterminants pour commencer à parler et à problématiser ce qui devient les littératures latino-américaines francophones ;

2 - la littérature faite par (et pas forcément pour) les femmes semble être une nouvelle aile qui se consolide dans ces études littéraires, dans lesquelles on constate que la première vague de penseurs et d'écrivains est encore des hommes (une donnée universellement attestée, sous quelque latitude qu'on soit !);

3 -le biais comparatif entre les littératures elles-mêmes et d'elles avec d'autres littératures nationales et régionales est un énorme terrain en friche : il semble être un chemin actuel qui se raffermira; plusieurs articles augurent de cette «piste» de recherche;

4 - un vide flagrant quant à la littérature guyanaise. 
À la lecture de ces perspectives, les littératures francophones d'Amérique latine offrent encore un grand chemin à être exploré par des chercheurs latino-américains et brésiliens.

Nous tenons à remercier toute l'équipe de la revue Caligrama : Revista de Estudos Românicos, et surtout sa rédactrice en chef Aléxia Teles pour nous avoir accueillis dans ce numéro.

À toutes et à tous, que ces lectures accélèrent la fin du confinement planétaire.

\section{Références bibliographiques}

CASANOVA, P. Paris, méridien de Greenwich de la littérature. In: CHARLE, C.; ROCHE, D. (dir.). Capitales culturelles, capitales symboliques : Paris et les expériences européennes (XVIIIe-XXe siècles). Paris: Éd. de la Sorbonne, 2002. p. 289-296.

DAMAS, L-G. Retour de Guyane. Paris: José Corti, 1938 .[Réédition 2003].

DAMAS, L.-G. «Afro-America », New Sum of Poetry from the Negro World. Présence Africaine, [S.l.], v. 59, p. 1-354, 1966.

LE BRIS, M.; ROUAUD, J.; ALMASSY, E. Manifeste pour une littérature-monde. Paris : Gallimard, 2007.

MIGNOLO, W. La désobéissance épistémique. Rhétorique de la modernité, logique de la colonialité et grammaire de la décolonialité. Trad. M. Maesschalck. Berlin; New York: Peter Lang, 2015.

PASSA PORTA. Les présents de l'écriture, essais et propos par. Bruxelles : Les Impressions Nouvelles, 2015.

PAZ, O. La Quête du présent. Trad. J. C. Masson. Paris : Gallimard, 1991.

Dennys Silva-Reis (Université Fédérale d'Acre) Kathleen Gyssels (Université d'Anvers) 